CASTELO BRANCO, M.; FRANÇA, F.H.; PONTES, L.A.; AMARAL, P.S.T. Avaliação da suscetibilidade a inseticidas em populações de traça-dascrucíferas de algumas áreas do Brasil. Horticultura Brasileira, Brasília, v. 21, n. 3, p. 549-552, julho-setembro 2003.

\title{
Avaliação da suscetibilidade a inseticidas em populações da traça-das- crucíferas de algumas áreas do Brasil
}

\author{
Marina Castelo Branco $^{1}$; Félix H. França ${ }^{1}$; Ludmilla A. Pontes ${ }^{2}$; Pablo S.T. Amaral ${ }^{2}$ \\ ${ }^{1}$ Embrapa Hortaliças, C. Postal 218, 70359-970 Brasília-DF; E-mail: marina@cnph.embrapa.br; ${ }^{2}$ Estudantes de Graduação, Univ. \\ Católica de Brasília; Estagiários Embrapa Hortaliças
}

\section{RESUMO}

A traça-das-crucíferas (TDC) é a praga mais importante do repolho, sendo basicamente controlada por inseticidas. Em alguns casos, as lavouras são pulverizadas duas a quatro vezes por semana, sem sucesso. Isto acontece porque, em muitos casos, são empregados inseticidas ineficientes, para os quais a praga já apresenta resistência. Trabalhos anteriores demonstraram ser possível determinar os inseticidas ineficazes para o controle da TDC em testes de laboratório, através do uso da dosagem recomendada. Neste trabalho foram coletadas larvas e pupas do inseto nos estados do Ceará (Tianguá), Minas Gerais (Barroso), Bahia (Mucugê), Mato Grosso (Sinop) e no Distrito Federal (Brazlândia e Embrapa Hortaliças). As populações foram criadas em laboratório e, dependendo do número de larvas de primeira geração, estas foram tratadas com as dosagens recomendadas de abamectin, acefato, B. thuringiensis, cartap, clorfluazuron, deltametrina e spinosad. Foi previamente assumido que um inseticida eficiente seria aquele que causasse a mortalidade de mais de $90 \%$ das larvas. Os resultados mostraram que a eficiência dos inseticidas variou entre as diferentes áreas. Spinosad causou a mortalidade de $100 \%$ das larvas em todos os locais. Foram ineficientes acefato, $B$. thuringiensis e cartap em Tianguá; abamectin em Brazlândia e clorfluazuron em Mucugê. Deltametrina não foi eficiente no controle das populações da praga coletadas em nenhuma das áreas geográficas amostradas. Conclui-se que as populações de traça-das-crucíferas brasileiras são resistentes a um ou mais ingredientes ativos e programas de manejo de resistência a inseticidas em TDC devem ser implementados.

Palavras-chave: Plutella xylostella, repolho, controle químico, resistência a inseticidas.

\begin{abstract}
Forecasting insecticide susceptibility in Diamondback Moth populations from different areas of Brazil
\end{abstract}

The Diamondback Moth (DBM) is an important brassica pest and is controlled by several types of insecticides. On some occasions, the fields are sprayed two-four times per week without success. Sometimes this occurs because ineffective insecticides are used. Works showed that laboratory tests using the recommended field rate of insecticides can detect the ineffective ones. Here, we collected DBM larvae and pupae from the States of Ceará (Tianguá), Minas Gerais (Barroso), Bahia (Mucugê), Mato Grosso (Sinop) and the Federal District (Brazlândia and Embrapa Hortaliças). We reared the populations in the laboratory and larvae of the first laboratory generation were treated with the recommended field rate of abamectin, acephate, B. thuringiensis, cartap, chlorfluazuron, deltamethrin, and spinosad. We previously determined that an effective insecticide should cause more than $90 \%$ larval mortality. Insecticide effectiveness was different from field to field. Spinosad killed $100 \%$ of larvae; acephate, B. thuringiensis and cartap killed less than $90 \%$ of the larvae in Tianguá; abamectin and chlorfluazuron did not control the insect in Brazlândia and Mucugê respectively. Deltamethrin was inneffective in all areas tested. Diamondback Moth populations were resistant to one or more active ingredients and programmes to manage insecticide resistance must be implemented in Brazil.

Keywords: Plutella xylostella, cabbage, chemical control, insecticide resistance.

\section{(Recebido para publicação em 09 de setembro de 2002 e aceito em 30 de abril de 2003)}

$\mathrm{A}$ experiência do Distrito Federal tem demonstrado que em épocas de temperatura elevada e ausência de precipitação ocorrem os maiores problemas em lavouras de repolho devido aos danos da traça-das-crucíferas (TDC) (França et al., 1985). Além dos fatores ambientais que favorecem o rápido crescimento das populações do inseto (Barrantes \& Rodriguez, 1996), as práticas culturais utilizadas (plantios sucessivos e não eliminação de restos culturais) aumentam o potencial de dano da praga, devido à possibilidade de sua multiplicação contínua. Para reduzir os prejuízos, muitos produtores têm optado pelo método que, aparentemente, pode produzir os melhores resultados: aplicações intensivas de inseticidas para controle da TDC. No entanto, esta prática, ao longo do tempo, tem se mostrado inefetiva já que aplicações de inseticidas até três vezes por semana não reduziram os danos do inseto (Castelo Branco et al., 2001). Nas demais regiões produtoras de repolho do país, foi também observado que as perdas ocorrem nos períodos secos e quentes do ano; além do mais, a aplicação intensiva de inseticidas e as práticas culturais destes locais não se diferenciam das utilizadas no DF.

Tendo em vista os sérios problemas econômicos ocasionados pela TDC, é necessário que mudanças no sistema de produção de repolho sejam implementadas. Entre as principais podemos citar: eliminação de restos de cultura e intervalo entre os plantios visando reduzir a população local de TDC; uso de irrigação por aspersão para redução do número de larvas sobre as plantas (Oliveira et al., 2000), redução do número de aplicações de inseticidas, através do uso do nível de dano econômico (Carballo \& Hruska, 1989; Castelo Branco et al., 1996); utilização, quando necessário, de inseticidas eficientes.

Para identificação dos produtos eficientes para controle da TDC, Zhao \& Grafius (1993) e Zhao et al. (1995) re- 
Tabela 1. Inseticidas utilizados em áreas agrícolas de cinco estados brasileiros para controle da traça-das crucíferas.1999 a 2001 . Brasília, Embrapa Hortaliças, 2002.

\begin{tabular}{|c|c|c|c|c|c|c|}
\hline \multirow[b]{2}{*}{ Inseticida } & \multirow{2}{*}{$\begin{array}{l}\text { Grupo } \\
\text { Químico }\end{array}$} & \multicolumn{2}{|c|}{1999} & \multicolumn{2}{|c|}{2000} & \multirow{2}{*}{$\begin{array}{c}2001 \\
\text { Sinop } \\
\text { (MT) }\end{array}$} \\
\hline & & $\begin{array}{c}\text { Barroso } \\
\text { (MG) }\end{array}$ & $\begin{array}{c}\text { Tianguá } \\
\text { (CE) }^{2}\end{array}$ & $\begin{array}{c}\text { Brazlândia } \\
(\mathrm{DF})^{3}\end{array}$ & $\begin{array}{c}\text { Mucugê } \\
\text { (BA) }\end{array}$ & \\
\hline Abamectin & Avermectina & & & $x$ & $\mathrm{X}$ & \\
\hline Bacillus thuringiensis & Biológico & & & $x$ & $x$ & \\
\hline Clorfluazuron & Benzoiluréia1 & $x$ & & & $x$ & \\
\hline Deltametrina & Piretróide & $x$ & & & $x$ & $x$ \\
\hline Fempropatrina & Piretróide & $x$ & & & $x$ & \\
\hline Lufenuron & Aciluréia1 & & & & $x$ & \\
\hline Nuvacron & Fosforado & & & & & $x$ \\
\hline Metomil & Carbamato & $x$ & & & $x$ & \\
\hline Metamid ofós & Fosforado & & $x$ & & $x$ & $x$ \\
\hline Paration Metil & Fosforado & $x$ & & & $x$ & \\
\hline Teflubenzuron & Fosforado & $x$ & $x$ & & $x$ & \\
\hline
\end{tabular}

${ }^{1 /}$ Regulador de crescimento; ${ }^{2 /}$ Inseticidas usados em mistura; ${ }^{3 /}$ Inseticidas alternados semanalmente

comendaram a realização de testes de laboratório onde larvas do inseto se alimentam com folhas de repolho tratadas com a dosagem recomendada do inseticida. Sannaveerappanavar \& Viraktamath (1997), Castelo Branco (1998) e Castelo Branco \& França (2000) empregaram este método e constataram que os inseticidas eficientes em laboratório foram eficientes também no campo.

Desse modo, levando-se em conta que este procedimento pode ajudar agricultores e extensionistas a selecionarem os inseticidas apropriados para sua região, este trabalho teve como objetivo avaliar a eficiência da dosagem comercial de diversos inseticidas para o controle de populações da TDC coletadas em diferentes regiões brasileiras.

\section{MATERIAL E MÉTODOS}

Populações de TDC foram coletadas em 1999 em Tianguá (CE) e Barroso (MG), em 2000 em Brazlândia (DF), Embrapa Hortaliças (DF) e Mucugê (BA) e em 2001 em Sinop (MT). Os produtores informaram os inseticidas utilizados (Tabela 1) e o número de aplicações destes produtos variou de uma (Tianguá, Brazlândia e Sinop) a duas por semana (Barroso e Mucugê). Não foram utilizados inseticidas na área $\mathrm{da}$ Embrapa Hortaliças.
As populações foram trazidas para o laboratório de Entomologia da Embrapa Hortaliças e criadas sobre folhas de repolho. Larvas de segundo estádio da primeira geração foram utilizadas nos testes de laboratório. O número de inseticidas avaliados para cada população dependeu do número de larvas disponíveis. Para cada teste, discos de folha de repolho com $4 \mathrm{~cm}$ de diâmetro foram tratados com as dosagens recomendadas dos inseticidas (g.i.a./ha): abamectin (9), acefato (562,5), Bacillus thuringiensis (18), cartap (300), clorfluazuron (25), deltametrina (6), spinosad $(38,4)$. Os inseticidas foram diluídos tomando-se como padrão um volume de calda de $400 \mathrm{~L} / \mathrm{ha}$. Espalhante adesivo (Agral $\left.{ }^{\circledR} 2 \mathrm{~mL} / \mathrm{L}\right)$ foi adicionado a todos os tratamentos. Em todos os experimentos foi utilizada uma testemunha tratada com água e espalhante.

Após o preparo das soluções, os discos de folha de repolho foram imersos nas soluções por cerca de $10 \mathrm{~s}$ e após a eliminação do excesso de umidade, estes foram colocados em placas de Petri de $9 \mathrm{~cm}$ de diâmetro e sobre cada disco foram colocadas 10 larvas. Foram utilizadas quatro repetições por tratamento. A mortalidade de larvas foi avaliada após $48 \mathrm{~h}$ para acefato e deltametrina; após $72 \mathrm{~h}$ para abamectin, $B$. thuringiensis e spinosad, porque estes inseticidas apresentam uma ação mais lenta sobre as larvas. Para clorfluazuron a mortalidade foi avaliada até que as pupas estivessem formadas, já que estes inseticidas atuam sobre os vários estágios do inseto (França \& Castelo Branco, 1996). Todos os experimentos foram realizados a temperatura de $20^{\circ} \mathrm{C}$ e fotofase de $13 \mathrm{~h}$ e umidade de 70 $80 \%$. Os dados da percentagem de mortalidade foram corrigidos pela fórmula de Abbott (Abbott, 1925) e submetidos a análise de variância. Foi utilizado o teste da diferença mínima significativa (DMS) a 5\% de probabilidade para a separação de médias. Foram considerados eficientes os produtos onde a mortalidade de larvas foi superior a $90 \%$.

\section{RESULTADOS E DISCUSSÃO}

O piretróide deltametrina não controlou a traça-das-crucíferas de nenhuma das regiões, já que a mortalidade de larvas variou de 2 a $41 \%$ (Tabela 2). Este inseticida começou a ser utilizado no Brasil no início da década de 80 e até o início dos anos 90, o produto apresentava eficiência (França et al., 1985; Silva et al., 1993; Villas Bôas et al., 1990). Em alguns locais esta eficiência foi atribuída à descontinuidade de seu uso, possibilitando a sobrevivência de individuos suscetíveis ao inseticida (Villas Bôas et al., 1990). No entanto, 
Tabela 2. Mortalidade de larvas de traça-das-crucíferas provenientes de cinco estados brasileiros em função da dosagem comercial de inseticidas ${ }^{1}$. $\mathrm{T}=20^{\circ} \mathrm{C}$, fotofase $14 \mathrm{~h}, \mathrm{UR}=70-80 \%$. Brasília, Embrapa Hortaliças, 2002.

\begin{tabular}{|c|c|c|c|c|c|c|c|}
\hline \multirow[b]{3}{*}{ Inseticida } & \multirow{3}{*}{$\begin{array}{c}\text { Dose } \\
\text { (g i.a./ha) }\end{array}$} & \multicolumn{6}{|c|}{$\%$ de mortalidade de larvas (média \pm EPM) } \\
\hline & & \multicolumn{2}{|c|}{1999} & \multicolumn{3}{|c|}{2000} & \multirow{2}{*}{$\begin{array}{l}2001 \\
\text { Sinop } \\
(M S)\end{array}$} \\
\hline & & $\begin{array}{c}\text { Barroso } \\
\text { (MG) }\end{array}$ & $\begin{array}{l}\text { Tianguá } \\
\text { (CE) }\end{array}$ & $\begin{array}{l}\text { Brazlândia } \\
\text { (DF) }\end{array}$ & $\begin{array}{c}\text { Embrapa } \\
\text { Hortaliças } \\
\text { (DF) }\end{array}$ & $\begin{array}{c}\text { Mucugê } \\
\text { (BA) }\end{array}$ & \\
\hline Abamectin & 9 & $92,5 \pm 4,7 a$ & $100,0 \pm 0,0 a$ & $87,2 \pm 5,4 \quad b$ & $92,6 \pm 7,3 a$ & $100,0 \pm 0,0 a$ & $100,0 \pm 0,0 a$ \\
\hline $\begin{array}{l}\text { Bacillus } \\
\text { thuringiensis }\end{array}$ & 18 & $100,0 \pm 0,0 a$ & $75,0 \pm 6,4 \quad b c$ & $100,0 \pm 0,0 a$ & $100,0 \pm 0,0 a$ & $100,0 \pm 0,0 a$ & $100,0 \pm 0,0 a$ \\
\hline Cartap & 300 & $100,0 \pm 0,0 a$ & $89,5 \pm 6,0 \quad b$ & $97,2 \pm 2,7 a$ & $93,7 \pm 3,7 a$ & $100,0 \pm 0,0 a$ & $100,0 \pm 0,0 a$ \\
\hline Clorfluazuron & 25 & $95,0 \pm 2,8 a$ & $97,5 \pm 2,5 a$ & --- & --- & $65,2 \pm 15,2 b$ & -- \\
\hline Spinosad & 38,4 & --- & --- & $100,0 \pm 0,0 a^{7}$ & $100,0 \pm 0,0 a$ & --- & $100,0 \pm 0,0 a$ \\
\hline Acefato & 562,5 & $95,0 \pm 2,8 a$ & $77,9 \pm 2,6 \quad b c$ & --- & --- & $91,0 \pm 5,5 a$ & $100,0 \pm 0,0 a$ \\
\hline Deltametrina & 6 & $2,2 \pm 2,2 b$ & $4,7 \pm 2,3$ & $13,9 \pm 5,7$ & $27,5 \pm 7,8 \quad b$ & $41,1 \pm 7,3 \mathrm{~b}$ & $15,0 \pm 5,0 b$ \\
\hline C.V. (\%) & & 14,3 & 14,7 & 13,9 & 19,8 & 9,8 & 3,9 \\
\hline
\end{tabular}

Médias seguidas de mesma letra, na mesma coluna, não diferem entre si pelo teste DMS ( $>0,05)$

${ }^{1 /}$ Testes realizados com larvas da primeira geração obtidas em criação de laboratório

no final dos anos 90, populações resistentes a deltametrina foram encontradas no DF. Para a população mais resistente ao inseticida, a dosagem que causava a mortalidade de $50 \%$ da população era de $73 \mathrm{~g}$ i.a./ha, ou seja, 12 vezes a dose recomendada de $6 \mathrm{~g}$ i.a./ha (Castelo Branco \& Gatehouse, 1997). Os resultados indicaram que, após o uso intensivo de deltametrina, o inseticida já não é eficente em diversos locais do país (Tabela 2), sugerindo que, pela mortalidade obtida, o nível de resistência destas populações é bastante elevado. Assim, seria conveniente que deltametrina tivesse o seu uso restringido.

Ainda com relação a deltametrina, é sabido que os mecanismos que fazem com que o inseto sobreviva às aplicações deste piretróide, são os mesmos que permitem a sobrevivência da TDC a outros produtos do mesmo grupo químico (Cheng, 1986). Desse modo, piretróides como fenvalerato, permetrina ou cipermetrina devem ter também o seu uso restringido.

Com relação aos demais inseticidas, foi observado que, em Mucugê, apenas o regulador de crescimento clorfluazuron foi ineficiente, em Brazlândia, apenas abamectin e em Tianguá, o fosforado acefato (Tabela 2). Neste locais, os produtos, ou outros do mesmo grupo químico como o fosforado metamidofós em Tianguá, já vinham sendo utilizados há mais de seis meses, o que indica que indivíduos capazes de sobreviver à dosagem recomendada dos inseticidas devem ter sido selecionados ao longo do período de utilização dos produtos.

Em Tianguá, onde $B$. thuringiensis e Cartap não eram utilizados, foi observado que os produtos não eram eficientes para o controle da TDC (Tabela 2). Uma das hipóteses para explicar esses resultados poderia ser a imigração e multiplicação de indivíduos capazes de sobreviver à dosagem comercial dos produtos, provenientes de áreas próximas ou distantes e o lento declínio da resistência em ausência de aplicações de inseticidas.

A movimentação da TDC entre diferentes áreas de cultivo foi observada por Honda et al. (1992) e Saito et al. (1998). Caprio \& Tabashnik (1992) verificaram que, quando insetos resistentes migram para áreas de cultivo, e a resistência é dominante, o nível de resistência das populações que recebem estes imigrantes aumenta. Já foi constatado que a resistência a cartap e $B$. thuringiensis é dominante (Liu \& Tabashnik, 1997, Cheng et al., 1999, Sayyed \& Wright, 2001). Aliado a isto, Hama (1990), Hama et al. (1992) e Murai et al. (1992) verificaram que ocorre lento declínio no nível de resistência a estes dois inseticidas em populações não submetidas a aplicações do produto.
Spinosad, um inseticida recém-lançado no mercado, foi eficiente para controle da TDC, causando a mortalidade de $100 \%$ das larvas tratadas (Tabela 2). $\mathrm{Na}$ China, larvas tratadas com a dosagem recomendada do inseticida também apresentaram o mesmo percentual de mortalidade, indicando eficiência do produto (Zhang et al., 2001). Testes preliminares de laboratório para avaliar a susceptibilidade de uma população coletada na Embrapa Hortaliças em uma área onde Spinosad nunca havia sido usado, mostraram que a população era altamente suscetível ao produto; a dosagem equivalente a $3,8 \mathrm{~g}$ i.a./ha (10 vezes menos que a dosagem comercial), ocasionava a mortalidade de $100 \%$ da população (Castelo Branco, dados não publicados).

Os resultados aqui obtidos confirmam que bioensaios de laboratório podem ser de grande utilidade para identificação de inseticidas eficientes para o controle da TDC (Sannaveerappanavar \& Viraktamath, 1997; Castelo Branco, 1998; Castelo Branco \& França, 2000), e podem contribuir, aliado a outras práticas, para reduzir as perdas econômicas dos agricultores e os problemas de poluição ambiental decorrentes do uso indiscriminado destes produtos.

Por fim, vale ressaltar que o método aqui empregado, avaliação da eficiência da dosagem recomendada de um inseticida, identifica os inseticidas que ti- 
veram a sua eficiência reduzida após uso prolongado, ou seja, aqueles para os quais as populações resistentes já foram selecionadas (Sereda et al., 1997). Isto traz prejuízos econômicos para as indústrias que comercializam estes produtos e limita os agrotóxicos disponíveis para os agricultores. Por isso, é necessária a implementação de programas de manejo de resistência a inseticidas em brássicas nas diversas regiões brasileiras. Estes programas devem incluir redução do número de aplicações de inseticidas, onde os produtos deve ser empregados apenas quando a praga atingir o nível de dano econômico e também promover a rotação de inseticidas (Castelo Branco \& França, 2000).

\section{AGRADECIMENTOS}

Aos Drs. Geni L. Villas Bôas e Maria Alice de Medeiros pela revisão do manuscrito. A Hozanan P. Chaves pelo auxílio nos trabalhos de campo.

\section{LITERATURA CITADA}

ABBOTT, W.S. A method of computing the effectiveness of an insecticide. Journal of Economic Entomology, v. 18, p. 265-267, 1925.

BARRANTES, A.J.A.; RODRIGUEZ, V.C.L. Abundancia estacional y dano de Plutella xylostella (L.) (Lepidoptera: Plutellidae) y el cultivo de repollo, durante la epoca seca en Alfaro Ruiz, Alajuela, Costa Rica. Manejo Integrado de Plagas, v. 39 , p. 17-24, 1996.

CAPRIO, M.A.; TABASHNIK, B.E. Gene flow accelerates local adaptation among finit populations: simulating the evolution of insecticide resistance. Journal of Economic Entomology, v. 85, p. 611-620, 1992.

CARBALLO, V.M.; HRUSKA, A.J. Periodos criticos de proteccion y efecto de la infestacion de Plutella xylostella L. (Lepidoptera: Plutellidae) sobre el rendimiento del repollo. Manejo Integrado de Plagas, v. 14 , p. 46-60, 1989.

CASTELO BRANCO, M. Inseticidas para o controle da traça-das-crucíferas: avaliação da eficiência, resistência e impacto sobre inimigos naturais. Embrapa Hortaliças, Brasília. (Boletim de Pesquisa da Embrapa Hortaliças 1). 1998.
CASTELO BRANCO, M.; FRANÇA, F.H. Previsão da eficiência de inseticidas para o controle da traça-das-crucíferas através do uso de doses discriminantes. Embrapa Hortaliças, Brasília. (Boletim de Pesquisa 2 da Embrapa Hortaliças). 2000. CASTELO BRANCO, M.; GATEHOUSE, A.G. Insecticide resistance in Plutella xylostella (L.) (Lepidoptera: Yponomeutidae) in the Federal District, Brazil. Anais da Sociedade Entomológica do Brasil, v. 26, p. 75-79, 1997.

CASTELO BRANCO, M.; VILLAS BÔAS, G.L.; FRANÇA, F.H. Nível de dano de traça das crucíferas em repolho. Horticultura Brasileira, Brasília, v. 14, p. 154-157, 1996.

CASTELO BRANCO, M.; FRANÇA, F.H.; MEDEIROS, M.A.; LEAL, J.G.T. Uso de inseticidas para o controle da traça-do-tomateiro e traça-das-crucíferas: um estudo de caso. Horticultura Brasileira, Brasília, v. 19, p. 60-63, 2001.

CHENG, E.Y. The resistance, cross-resistance and chemical control of Diamondback Moth in Taiwan. In: Talekar, N.S.; Griggs, T.D. Proceedings of the First International Workshop. AVRDC. Shanhua, Taiwan. p. 329-345. 1986.

CHENG, L.G.; LI, F.L.; CHEN, Z.H.; WANG, Y.C. Genetic analysis of cartap resistance in diamondback moth, Plutella xylostella (L.). Acta Entomologica Sinica, v. 42, p. 12-18, 1999.

FRANÇA, F.H.; CASTELO BRANCO, M. Controle de pragas de hortaliças com produtos reguladores de crescimento de inseto. Horticultura Brasileira, Brasília, v. 14, p. 4-8, 1996.

FRANÇA, F.H.; CORDEIRO, C.M.T.; GIORDANO, L.B.; RESENDE, A.M. Controle da traça-das-crucíferas em repolho, 1984. Horticultura Brasileira, Brasília, v. 3, n. 2, p. 47 53. 1985.

HAMA, H. Insecticide resistance of diamondback moth, Plutella xylostella in Japan. Japan Agricultural Research Quarterly, v. 24, p. 22-30, 1990.

HAMA, H.; SUZUKI, K.; TANAKA, H. Inheritance and stability of resistance to Bacillus thuringiensis formulations of the diamondback moth, Plutella xylostella (Linnaeus) (Lepidoptera: Yponomeutidae). Applied Entomology and Zoology, v. 27, p. 355-362, 1992.

HONDA, K.; MIYAHARA, Y.; KEGASAWA, K. Seasonal abundance and the possibility of spring immigration of the diamondback moth, Plutella xylostella (Linnaeus) (Lepidoptera: Yponomeutidae), in Morioka City, Northern Japan. Applied Entomology and Zoology, v. 27, p. 517525, 1992.

LIU, Y.B.; TABASHNIK, B.E. Inheritance of resistance to the Bacillus thuringiensis toxin Cry $1 \mathrm{C}$ in the diamondback moth. Applied and Environmental Microbiology, v. 63, p. 2218-2223, 1997.
MURAI, T.; MIYAZAKI, M.; OZUKA, M. Changes in insecticide susceptibility of the Diamondback Moth in Shimane, Japan. Japan Agricultural Research Quarterly, v. 26, p. 152156, 1992.

OLIVEIRA, A.T.;JUNQUEIRA, A.M.R.; FRANÇA, F.H. Impacto da irrigação por aspersão convencional na dinâmica populacional da traça-dascrucíferas em plantas de repolho. Horticultura Brasileira, Brasília, v. 18, p. 37-40, 2000.

SANNAVEERAPPANAVAR, V.T.; VIRAKTAMATH, C.A.: Management of insecticide resistant diamondback moth, Plutella xylostella L. (Lepidoptera: Yponomeutidae) on cabbage using some novel insecticides. Mysore Journal of Agricultural Sciences, v. 31, p. 230235, 1997.

SAITO, O.; MIZUSHIMA, S.; OKUYAMA, S.; HANADA, T.; TORIKURA, H.; HACHIYA, K.; SATO, K. Biology of the diamondback moth, Plutella xylostella (L.), in Hokkaido. Research Bulletin of the Hokkaido National Agricultural Experiment Station, v. 167, p. 69-110, 1998.

SAYYED, A.H.; WRIGHT, D.J. Cross-resistance and inheritance of resistance to Bacillus thuringiensis toxin Cry1 Ac in diamondback (Plutella xylostella L) from lowland Malaysia. Pest Management Science, v. 57, p. 413-421, 2001. SEREDA, B.; BASSON, N.C.I.; MARAIS, P. Bioassay of insecticide resistance in Plutella xylostella (L.) in South Africa. African Plant Protection, v. 3, p. 67-72, 1997.

SILVA, A.L.; VELOSO, V.R.S.; TARDIVO, J.C.; ABREU, C.D.; SILVA, R.M.C. Avaliação de inseticidas piretróides no controle da traça-dascrucíferas Plutella xylostella (L., 1758) em repo1ho. Anais das Escolas de Agronomia e Veterinária, v. 23, n. 1, p. 7-12, 1993.

VILLAS BÔAS, G.L.; CASTELO BRANCO, M.; GUIMARÃES, A.L. Controle químico da traçadas-crucíferas em repolho no Distrito Federal. Horticultura Brasileira, Brasília, v. 8, n. 2, p. 1011, 1990.

ZHANG, X.Y.; HE, J.; YE, C.Y; XUE, Y. Monitoring on resistance of diamond back moth to abamectin and field control experiments in Yunnan, China. Journal of Huazhong Agricultural University. v. 20, p. 426-430, 2001.

ZHAO, J.Z.; GRAFIUS, E. Assessment of different bioassay techniques for resistance monitoring in the the Diamondback Moth (Lepidoptera: Plutellidae). Journal of Economic Entomology, v. 86, p. 995-1000, 1993.

ZHAO, J.Z.; WU, S.C.; ZHU, G.R. Bioassays with the recommended field concentrations of several insecticides for resistance monitoring in Plutella xylostella. Resistant Pest Management, v. 7, p. 1314, 1995. 